\title{
Reliability and Validity of a Free Oral Pre-exposure Prophylaxis (PrEP) Adherence Questionnaire Among Patients with Sexually Transmitted Infection and Without HIV Infection in Shanghai
}

\section{Xiaoyue Yu}

Shanghai Jiao Tong University School of Medicine,Shanghai,China

\section{Chen Xu}

School of Public Health,Shanghai Jiao Tong University School of Medicine,Shanghai,China

\section{Yang Ni}

School of Public Health,Shanghai Jiao Tong University School of Medicine,Shanghai,China

\section{Ruijie Chang}

School pf Public Health,Shanghai Jiao Tong University School of Medicine,Shanghai,China

Huwen Wang

School of Public Health,Shanghai Jiao Tong University School of Medicine,Shanghai,China

\section{Ruijie Gong}

School of Pubilv Health,Shanghai Jiao Tong University School of Medicine

\section{Ying Wang}

School of Public Health,Shanghai Jiao Tong University School of Medicine,Shanghai,China

\section{Suping Wang}

School of Public Health,Shanghai Jiao Tong University School of Medicine,Shanghai,China

\section{Yong Cai ( $\nabla$ caiyong202028@hotmail.com )}

School of Public Health, Shanghai Jiao Tong University School of Medicine https://orcid.org/00000002-5706-7906

\section{Research article}

Keywords: reliability and validity, pre-exposure prophylaxis (PrEP), HIV prevention, questionnaire, adherence

Posted Date: October 5th, 2020

DOI: https://doi.org/10.21203/rs.3.rs-73648/v1

License: (c) (1) This work is licensed under a Creative Commons Attribution 4.0 International License. Read Full License 
Page $2 / 20$ 


\section{Abstract}

Objective: In this study, we aimed to evaluate the validity and reliability of the free oral pre-exposure prophylaxis (PrEP) adherence questionnaire among patients with sexually transmitted infection who do not have HIV infection in Shanghai.

Methods: We conducted a cross-sectional study among 816 patients from sexually transmitted infection (STI) clinics in Shanghai. The questionnaire included self-reported demographic characteristics, items on adherence to free oral pre-exposure prophylaxis (PrEP), and PrEP uptake behavior measurement. Item analysis was carried out by calculating the critical ratio and item-total correlations. Internal consistency and split-half reliability were measured to assess reliability. Content validity, construct validity, convergent validity and discriminant validity were measured to assess validity. We conducted logistic regression with receiver operating characteristic (ROC) curve analysis to assess the predictive value of the adherence questionnaire for PrEP uptake behavior.

Results: All items were considered acceptable in the item analysis $(\mathrm{P}<0.05)$. The questionnaire had a Cronbach's alpha of 0.757 and split-half reliability of 0.633 . The scale-level content validity index (CVI) was 0.938 and the item-level CVI of each item ranged from 0.750 to 1 . In exploratory factor analysis, we introduced a four-factor model accounting for $79.838 \%$ of the aggregate variance with all factors loading more than 0.4 , which was validated in confirmatory factor analysis $\left(c^{2}=47.1\right.$, degrees of freedom $=14$, root mean square error of approximation $=0.078$ ). According to regression analysis, adding PrEP adherence questionnaire scores contributed to prediction of PrEP uptake behavior $(P<0.001)$. The maximum area under the ROC curve was 0.778 (95\% confidence interval: $0.739-0.817)$, corresponding to a cutoff score of 9 .

Conclusion: The PrEP adherence questionnaire generally has acceptable reliability and validity. This questionnaire can be used as a tool to evaluate PrEP adherence in patients with STI who do not have HIV infection.

\section{Introduction}

Since acquired immunodeficiency syndrome (AIDS) was first reported in 1981, it has quickly become a global epidemic. Knowledge of the epidemiological characteristics of AIDS has evolved considerably. Populations at high risk for acquiring human immunodeficiency virus (HIV) infection have shifted to sexually active groups, including people with sexually transmitted infection (STI). ${ }^{1,2}$ Whereas universal antiretroviral therapy for people living with HIV is recommended and does reduce mortality, it is a treatment and not a cure. ${ }^{3}$ Fortunately, the antiretroviral drug oral emtricitabine/tenofovir has been introduced as pre-exposure prophylaxis (PrEP), to help reduce HIV transmission.

Before the advent of PrEP, public health measures to prevent HIV infection such as condom use, antiretroviral therapy, male circumcision, and regular HIV testing of high-risk populations achieved great 
success in preventing HIV transmission. ${ }^{1}$ However, the development of PrEP has brought AIDS prevention to another level, and its efficiency and safety has been proven in many clinical trials. ${ }^{4-7}$ The initial clinical trials of PrEP have yielded different estimations of efficacy, which may be explained by varying population adherence. Two studies failed to demonstrate efficacy of PrEP, the Vaginal and Oral Interventions to Control the Epidemic (VOICE) trial with 29\% population adherence, and the Preexposure Prophylaxis Trial for HIV Prevention among African Women (FEM-PrEP) trial with 37\% population adherence. ${ }^{8,9}$ Compared with other clinical trials, such as the Preexposure Prophylaxis Initiative (iPrEx) trial and the Partners Preexposure Prophylaxis (Partner PrEP) Study, where population adherence was above $50 \%$, a low degree if adherence without efficient measures may explain the findings of the VOICE and FEM-PrEP trials. ${ }^{4,10}$ Nevertheless, drug adherence has an important role in the effectiveness of PrEP among people at high risk for HIV infection. ${ }^{11,12}$ Various measures have been introduced in recent clinical studies to improve adherence, such as use of short message service, drug level testing. and electronic medicine containers with automated monitoring that remind patients to take their medication. ${ }^{13}$ Nevertheless, unlike clinical trials in which drug adherence can be monitored and a more supportive environment is provided by researchers, medication adherence is difficult to predict; however, optimizing adherence is closely tied to PrEP efficacy. It is important to investigate how best to optimize adherence for populations with a high risk of acquiring HIV and to explore individual-level measures to ensure medication adherence. Simple, effective, user-friendly methods, such as questionnaires, are needed to evaluate individual adherence. In our previous study among transgender women sex workers, we assessed the acceptability of daily use of free PrEP, based on the theory of planned behavior; this formed the basis of a questionnaire developed to evaluate PrEP adherence. ${ }^{14}$

Whereas many studies have assessed PrEP uptake and adherence as well as the sustainability of providing PrEP services for men who have sex with men or transgender women, patients with STI require equivalent attention, as an identifiable population with a high-risk of acquiring HIV infection. ${ }^{10,14-22}$ In research by Galvin, the presence of an STI could increase the infectivity and susceptibility of HIV via various biological mechanisms, causing patients with STI to be more vulnerable to HIV infection. ${ }^{23}$ In a national review of the HIV infection epidemic trend in China, although the incidence of HIV infection was low among patients with STI, more HIV infections have been caused by heterosexual contact. ${ }^{2}$ Great benefits can be derived from promoting the use of PrEP and improving medication adherence among patients who are vulnerable to becoming infected with HIV.

Although the AIDS epidemic has remained at a low level in China, the overall incidence of AIDS is showing a gradual upward trend. ${ }^{2,24}$ What's more, in light of the three UNAIDS targets for AIDS prevention and control by 2020, ${ }^{25}$ improvement is needed in China, for which PrEP is a powerful resource. Therefore, the risk of HIV infection among patients with STI and without HIV infection should not be ignored. However, there is much uncertainty related to PrEP medication adherence among patients with STI. In this study, we aimed to evaluate the reliability and validity of a free oral PrEP adherence questionnaire for assessing PrEP adherence among patients with STI, and to explore the predictive accuracy of the 
questionnaire. Our findings can provide suggestions for future improvements in the prevention of HIV infection among the population with STI and other populations with greater risk of HIV infection.

\section{Methods}

\section{Participants}

We recruited outpatients and inpatients who visited STI clinics on Wednesdays and Saturdays at two branch institutes of Shanghai Dermatology Hospital in the Jingan district (located on Qiujiang Road and Baode Road, respectively) from November 2017 to May 2018.

The inclusion criteria were according to the latest revised STI prevention and management measures in China, and included patients aged over 16 years old with syphilis, gonorrhea, condyloma acuminatum, and genital herpes. All diagnoses (including clinical and laboratory diagnoses) were conducted by doctors in the hospital.

The exclusion criteria were as follows: (1) infected with HIV (2) serious psychological or cognitive diseases; (3) unconscious status; (4) unwilling to cooperate with researchers; (5) vision or hearing loss or poor reading ability, leading to little understanding of the purpose and content of this research.

\section{Data collection}

Our research team recruited all physicians working at STI clinics in two cooperative Shanghai Dermatology Hospitals. The researchers were in charge of training all doctors in how to select patients who met the inclusion and the exclusion criteria of this study. After training, doctors were responsible for informing eligible patients about the purpose and content of this research and determining their willingness to participate. The interviewer team comprised senior undergraduate or graduate students recruited from Shanghai Jiao Tong university school of medicine who had experience with administering questionnaire surveys. The interviewer team was responsible for conducting face-to-face interviews with patients. All interviews were conducted twice a week over nearly 7 months.

In each interview, one member of the interviewer team invited patients one by one to a quiet room, so as to keep patients focused on completing the questionnaire. The interview comprised three phases; during the first phase, patients were provided with an informed consent form to sign. Interviewers answered all patients' concerns in principle. During the second phase, patients were required to complete the questionnaire on their own; no private information was collected during this process. In the third phase, interviewers verified the completed questionnaire to ensure no items were missed or ignored. At the end of the interview, patients receive a cash stipend ( 80 RMB, nearly 12 USD) in consideration of their cooperation in this study. 


\section{Measurement}

The questionnaire included self-reported demographic characteristics, free oral PrEP adherence questionnaire, and PrEP uptake behavior items, to evaluate patients' adherence to PrEP ${ }^{14}$. Free oral PrEP adherence questionnaire used in this research is original, and eight items were included, based on factors found to be associated with the acceptability of free oral PrEP in our previous team work. ${ }^{14}$ Those eight items were used to measure participants' attitudes, subjective norms, and perceived behavioral control. Behavioral intention was measured using an additional question separate from the questionnaire, to predict the actual performance of each individual. Considering the conciseness and clarity of the questionnaire items, four subscales were established: benefits, barriers, peer support, and self-efficacy. Eight items were included, with two items for each subscale. Each item on the questionnaire was scored using a three-point scale: 0 points for "disagree", 1 point for "unclear", and 2 points for "agree"; the total score on the questionnaire was 16 points. Higher scores indicated higher adherence to PrEP. Both positive and negative questions were included, to reduce participants' response bias to a certain extent. (full questionnaire was shown in additional file 1)

Participants were asked the following question apart from the adherence questionnaire: Would you be willing to take PrEP on a daily basis during the following 6 months? There were five responses, corresponding to different degrees of PrEP uptake willingness or behavioral intention (answers: $1=$ definitely not, 2 = unlikely, 3 = neutral, $4=$ likely, 5 = definitely will). Given that not all behavioral intentions translate into action, ${ }^{26}$ we selected "definitely will" as the standard predicting positive behavior, in a conservative manner.

\section{Statistical analysis}

For item analysis, we used the critical ratio and Spearman correlation coefficient to test and evaluate the relevance and reliability of all items. In the calculation of critical ratio, participants were divided into two groups based on their total adherence scores: a high-adherence group (participants ranked among the $27 \%$ with the highest scores) and a low-adherence group (participants ranked among the $27 \%$ with the lowest scores). We conducted a $t$-test for scores of each item in the high-and low-adherence groups. A Spearman correlation test was conducted between each item score and the total score.

For reliability analysis, Cronbach's alpha coefficient was used to evaluate the internal consistency of the questionnaire, and the Spearman-Brown split-half coefficient was used to evaluate reliability of the questionnaire.

A panel of eight experts specializing in population health and health behavior was invited to conduct the content validity analysis, scoring the relevance of each item to the corresponding content concept, the simplicity and clarity of each item, and the ambiguity of each item. In addition, the experts gave their 
opinions, based on a four-point scale, in evaluating the questionnaire using the content validity index (CVI), including the item-level CVI (I-CVI) and scale-level CVI (S-CVI);

For construct validity, factor analysis was performed. Samples were randomly divided into two groups. Group 1 (406 patients) was used for exploratory factor analysis (EFA) and Group 2 (410 patients) for confirmatory factor analysis (CFA). In the EFA, Bartlett's test of sphericity and the Kaiser-Meyer-Olkin (KMO) value were applied for eligibility; principal component analysis was adopted to extract common factors. In the CFA, the maximum likelihood method was used to verify the fitness of the initial model. Goodness-of-fit indices such as $c^{2}$, degrees of freedom (df), p-value, root mean square error of approximation (RMSEA), goodness-of-fit index (GFI), adjusted goodness-of-fit index (AGFI), and comparative fit index $(\mathrm{CFI})$ were adopted.

The value of average variance extracted (AVE) and construct reliability (CR) were used to evaluate convergent validity. We used the square root value of AVE and related analysis results to evaluate discriminant validity.

For predictive value and score translation, univariate logistic regression, hierarchical logistic regression, and receiver operating characteristic curve (ROC) analyses were carried out to assess the predictive value of adherence questionnaire scores in discriminating patients with positive behaviors (high-adherence group) and negative behaviors (low-adherence group), and to determine the cutoff score.

The data were analyzed using SPSS 25.0, AMOS 22.0, and MedCalc 19.0.

\section{Results}

\section{Participant demographic and adherence-related characteristics}

A total of 816 participants were recruited in this study. The mean age of participants was 38.56 years, standard deviation 13.00 years. The mean adherence score was 8.79 , standard deviation 2.61 . Among the 816 participants, 134 (16.42\%) were aware of PrEP as a preventive measure for AIDS, and 11 patients (1.35\%) had taken PrEP before the study. Table 1 shows participant demographic and adherence-related characteristics and adherence scores between the groups. The total adherence score was significantly different according to age, education level, marital status, income, sexual orientation, and knowledge about PrEP (Table 1). 
Table 1

Participant demographic and adherence-related characteristics

$\begin{array}{lll}\text { Characteristics } & \mathrm{n}(\%) & \text { Adherence } \\ & \text { Score } \\ & \text { (mean } \pm \text { SD) }\end{array}$

\section{Age}

$18-30$

$263(32.23 \%) \quad 9.42 \pm 2.56$

$31-60$

$480(58.82 \%) \quad 8.70 \pm 2.47$

$61-80$

$73(8.95 \%)$

$7.36 \pm 2.88$

$<0.001$

\section{Gender}

Male

384

$(47.06 \%)$

Female

432(52.94\%)

$8.84 \pm 2.60$

0.498

\section{Education}

Primary school and below

$35(4.29 \%) \quad 7.26 \pm 2.44$

Junior high school

$162(19.85 \%) \quad 8.44 \pm 2.78$

Senior high school

$186(22.79 \%) \quad 8.52 \pm 2.68$

College and above

433(53.06\%)

$9.15 \pm 2.46$

$<0.001$

\section{Marital Status}

Single

Married

Divorced

Widowed

Income

Below 3000 RMB

3001-6000 RMB

6001-12000 RMB

12001 and above RMB

\section{Sexual Orientation}

Heterosexual

Homosexual

\begin{tabular}{lll}
\hline $232(28.43 \%)$ & $9.36 \pm 2.40$ & \\
\hline $520(63.73 \%)$ & $8.67 \pm 2.52$ & \\
\hline $53(6.50 \%)$ & $7.62 \pm 3.49$ & \\
\hline $11(1.35 \%)$ & $8.00 \pm 2.76$ & $<0.001$ \\
& & \\
\hline $122(14.95 \%)$ & $8.08 \pm 2.67$ & \\
\hline $285(34.93 \%)$ & $8.71 \pm 2.81$ & \\
\hline $223(27.33 \%)$ & $9.01 \pm 2.39$ & \\
\hline $186(22.79 \%)$ & $9.12 \pm 2.39$ & 0.04 \\
\hline
\end{tabular}

$745(91.30 \%) \quad 8.78 \pm 2.63$

$28(3.43 \%) \quad 10.32 \pm 2.25$ 


\begin{tabular}{|llll|}
\hline Bisexual & $10(1.23 \%)$ & $9.60 \pm 2.32$ & \\
\hline Pansexual & $3(0.37 \%)$ & $8.33 \pm 0.58$ & \\
\hline Past PrEP usage history & $30(3.68 \%)$ & $7.73 \pm 2.15$ & 0.006 \\
Yes & & & \\
\hline No & $11(1.35 \%)$ & $8.00 \pm 2.68$ & \\
\hline Knowledge about PrEP & $805(98.65 \%)$ & $8.80 \pm 2.61$ & 0.35 \\
\hline Unknown & & & \\
\hline Information from website and new media & $682(83.58 \%)$ & $8.69 \pm 2.54$ & \\
\hline Information from healthcare workers & $78(9.56 \%)$ & $9.64 \pm 2.83$ & \\
\hline Information from sexual partners, friends and other & $33(4.04 \%)$ & $8.97 \pm 3.28$ & \\
acquaintances & $8(0.98 \%)$ & $8.75 \pm 1.75$ & \\
\hline Information from other sources & & & \\
\hline PrEP, pre-exposure prophylaxis. & $15(1.84 \%)$ & $8.53 \pm 2.80$ & \\
\hline
\end{tabular}

\section{Item analysis}

Except for item 3, all items had a critical ratio value higher than 3, with statistical significance. Except for items 3 and 4, all items had a Spearman correlation coefficient greater than 0.3, with statistical significance. Items 1,2 , and 5-8 were acceptable in the item analysis (Table 2). 
Table 2

Item analysis of the PrEP adherence questionnaire

\begin{tabular}{|llllll|}
\hline Item & score & $\mathrm{CR}$ & $\mathrm{P}$ & item-total correlation & $\mathrm{P}$ \\
\hline 1 & $2.25 \pm 0.593$ & 19.023 & $<0.001$ & 0.674 & $<0.001$ \\
\hline 2 & $2.28 \pm 0.630$ & 19.245 & $<0.001$ & 0.688 & $<0.001$ \\
\hline 3 & $2.07 \pm 0.598$ & 2.945 & 0.003 & 0.169 & $<0.001$ \\
\hline 4 & $2.19 \pm 0.648$ & 3.273 & 0.001 & 0.166 & $<0.001$ \\
\hline 5 & $1.98 \pm 0.496$ & 10.626 & $<0.001$ & 0.492 & $<0.001$ \\
\hline 6 & $2.02 \pm 0.540$ & 16.456 & $<0.001$ & 0.690 & $<0.001$ \\
\hline 7 & $2.21 \pm 0.662$ & 23.555 & $<0.001$ & 0.728 & $<0.001$ \\
\hline 8 & $2.31 \pm 0.682$ & 23.609 & $<0.001$ & 0.686 & $<0.001$ \\
\hline PrEP, pre-exposure prophylaxis; CR, critical ratio. & \\
\hline
\end{tabular}

\section{Reliability}

Cronbach's alpha of total PrEP adherence for internal consistency was 0.757 and the Spearman correlation coefficient of total PrEP adherence for split-half reliability was 0.633 , which were acceptable (Table 3).

Table 3

Reliability analysis of the PrEP adherence questionnaire

\begin{tabular}{|lll|}
\hline & Cronbach's alpha & Spearman-Brown Split-Halt \\
\hline Benefit & 0.833 & 0.834 \\
\hline Barrier & 0.505 & 0.506 \\
\hline Peer support & 0.649 & 0.651 \\
\hline Self-efficacy & 0.750 & 0.750 \\
\hline Total scale & 0.757 & 0.633 \\
\hline PrEP, pre-exposure prophylaxis.
\end{tabular}

\section{Validity}




\section{Content validity}

The S-CVI was 0.938 , and the I-CVI for each item ranged from 0.750 to 1 . In general, the content validity of the questionnaire was acceptable.

\section{Exploratory factor analysis (EFA)}

The KMO value was 0.729 , Bartlett's spherical test $\chi^{2}=1064.865$, $d f=28, P<0.001$, indicating that the questionnaire was suitable in the EFA.

Four common factors with eigenvalues greater than 1 were extracted and accounted for $79.838 \%$ of the aggregate variance, which was in accordance with our theoretical four-factor structure. No item loads were less than 0.4. Whereas item 6 loaded in two subscales, it loaded heavier in factor $3(0.728)$ than in factor 4 (0.455); therefore, item 6 was classified into factor 3 (Table 4).

Table 4

Exploratory factor analysis of the PrEP adherence questionnaire

\begin{tabular}{|lllll|}
\hline Item & Subscale1 & Subscale & Subscale & Subscale \\
\hline $\begin{array}{l}\text { 1. PrEP can effectively reduce your risk of HIV } \\
\text { infection. }\end{array}$ & 0.880 & & & \\
\hline $\begin{array}{l}\text { 2. This medication could reduce the risk of HIV } \\
\text { transmission to your partner. }\end{array}$ & 0.893 & & & \\
$\begin{array}{l}\text { 3. The side effects of PrEP can affect your daily } \\
\text { life. }\end{array}$ & 0.791 & & \\
$\begin{array}{l}\text { 4. My partner would think I do not trust him/her, if } \\
\text { they find me taking this medication. }\end{array}$ & 0.813 & & \\
\hline $\begin{array}{l}\text { 5. A lot of my friend would be willing to take PrEP. } \\
\text { 6. My partner is supportive for me to take PrEP. }\end{array}$ & & 0.877 & 0.455 \\
\hline $\begin{array}{l}\text { 7. You are confident to use free PrEP if you want } \\
\text { to. }\end{array}$ & & & 0.864 \\
\hline $\begin{array}{l}\text { 8. It is up to me whether to take PrEP if PrEP is } \\
\text { free to access. }\end{array}$ & & & \\
\hline PrEP, pre-exposure prophylaxis. & & & \\
\hline
\end{tabular}

\section{Confirmatory factor analysis (CFA)}


After EFA, a four-factor structural model was adopted for CFA, which included the following factors influencing PrEP adherence among target participants: benefits, barriers, peer support, and self-efficacy. An acceptable model fit resulted, as follows: $c^{2}=47.1, d f=14, c^{2} / d f=3.361$, RMSEA=0.078, GFI=0.975, AGFI=0.936, and CFI=0.962 (Table 5).

Table 5

Confirmatory factor analysis of the PrEP adherence questionnaire

\begin{tabular}{|c|c|c|c|c|c|c|}
\hline X2 & $d f$ & $\mathrm{X} 2 / \mathrm{df}$ & RMSEA & GFI & AGFI & CFI \\
\hline 47.1 & 14 & 3.361 & 0.076 & 0.975 & 0.936 & 0.962 \\
\hline
\end{tabular}

\section{Convergent and discriminant validity}

All subscales had a CR value and AVE value greater than 0.7 and 0.5 , respectively, except for subscale support; however, the CR value of subscale support was nearly 0.7 (0.691), so the convergent validity was acceptable. The square root AVE value of each subscale was greater than the maximum correlation coefficient between the subscale and other subscales; therefore, discriminant validity was acceptable (Tables 6 and 7).

Table 6

Convergent validity analysis of PrEP adherence questionnaire

\begin{tabular}{|lllllll|}
\hline Item & Subscale & Estimate & Standard Estimate & S.E. & CR & AVE \\
\hline 1 & Benefit & 1.000 & 0.861 & & & \\
\hline 2 & Benefit & 1.024 & 0.831 & 0.054 & 0.834 & 0.716 \\
\hline 3 & Barrier & 0.268 & 0.314 & & & \\
\hline 4 & Barrier & 1.000 & 1.079 & 0.204 & 0.725 & 0.631 \\
\hline 5 & Peer support & 1.000 & 0.542 & & & \\
\hline 6 & Peer support & 1.785 & 0.889 & 0.169 & 0.691 & 0.542 \\
\hline 7 & Self-efficacy & 1.085 & 0.820 & & & \\
\hline 8 & Self-efficacy & 1.000 & 0.733 & 0.065 & 0.753 & 0.605 \\
\hline $\begin{array}{l}\text { PrEP, pre-exposure prophylaxis; CR, construct reliability; S.E., standard error; AVE, average variance } \\
\text { extracted. }\end{array}$ & & & & & \\
\hline
\end{tabular}


Table 7

Discriminant validity analysis of PrEP adherence questionnaire

\begin{tabular}{|c|c|c|c|c|}
\hline & Self-efficacy & Peer support & Barrier & Benefit \\
\hline Self-efficacy & 0.778 & & & \\
\hline Peer support & 0.090 & 0.736 & & \\
\hline Barrier & 0.010 & 0.001 & 0.795 & \\
\hline Benefit & 0.157 & 0.072 & 0.018 & 0.846 \\
\hline
\end{tabular}

\section{Predictive value and score translation}

The score of the PrEP adherence questionnaire was a statistically significant predictor of PrEP uptake behavior in the initial univariate logistic regression analysis (odds ratio $=1.585, \mathrm{P}<0.001$ ). In hierarchical logistic regression, entry of the adherence questionnaire score improved prediction significantly $(P<0.001)$ (Table 8).

Table 8

Hierarchical regression analysis of PrEP adherence questionnaire score for predicting PrEP uptake behavior

\begin{tabular}{|llll|}
\hline predictive outcome & Variable added & Model change & P value \\
\hline PrEP uptake behavior $\quad$ total score & 110.4 & $<0.001$ \\
\hline PrEP, pre-exposure prophylaxis; & & \\
\hline
\end{tabular}

The ROC curve of the self-report PrEP adherence questionnaire score to determine participants' actual PrEP uptake behavior and the area under the ROC curve (AUC) suggested that the PrEP adherence questionnaire performs well in distinguishing patients with high adherence and low adherence. The maximum AUC value under the curve was 0.778 (95\% confidence interval: 0.739-0.817), corresponding to a cutoff score of 9 with a specificity of $72.24 \%$ and sensitivity of $74.39 \%$ (Figure 1 ).

\section{Discussion}

Since the introduction of PrEP as primary prevention for HIV transmission, its efficacy has been evident in rigorous clinical trials. ${ }^{4,10}$ Nevertheless, it is important to maximize the use of PrEP to best prevent HIV 
transmission. Adherence is one factor that has an impact on PrEP efficacy. ${ }^{1}$ The aim of this research was to assess the reliability and validity of the self-report PrEP adherence questionnaire among patients with STI who did not have but were at high risk of HIV infection. Our results demonstrated that the PrEP adherence questionnaire had acceptable reliability and validity and could serve as a useful measurement to evaluate differences in PrEP adherence among patients with STI.

We conducted item analysis and evaluated reliability and validity in this study. In item analysis, items 3 and 4 showed disqualifying results; however, the subscales of items 3 and 4 describe the factors that hinder adherence to PrEP, including drug side-effects and HIV stigma. The influence of these two factors on PrEP has been shown in previous studies. ${ }^{18,27,28}$ In Golub's research, PrEP stigma was found to be highly associated with HIV stigma as PrEP is designed to prevent HIV infection. ${ }^{28}$ People tend to relate HIV stigma to concerns about being seen taking medicine by family or friends, and such concerns substantially impact adherence. ${ }^{19,29}$ In our design of item 4, we mainly considered concerns from sexual partners, which could represent PrEP stigma to some extent. Moreover, the critical ratio value and correlation coefficient of items 3 and 4 were statistically significant; therefore, we retained those two items. The relatively lower critical ratio value and correlation coefficient of items 3 and 4 may result from the language or expressions used, which may require more specificity and clarity. Another issue that may account for this situation was that clinical side-effects and HIV stigma were not the main barriers to PrEP among patients with STI. Further investigation into barriers among the population with STI is required. In reliability analysis, results for internal consistency and external reliability showed that the questionnaire is sufficiently stable and has acceptable reliability. In validity analysis, we first carried out EFA. According to the questionnaire design, four common factors had to be extracted during this process. Through principal component analysis, four common factors with an aggregate variance contribution rate up to $79.838 \%$ were obtained, indicating that the variance caused by the four common factors can well explain variation in the variables measured in the questionnaire. In addition, the load of each item on the subscale to which it belongs was above 0.7 , demonstrating that the classification of each item to its corresponding subscale was consistent with our design purpose. Furthermore, CFA indicated that the theoretical model, divided according to the designed four subscales, could well explain the actual data; the above results with acceptable convergent and discriminant validity indicated that the questionnaire has good structural validity. Furthermore, the results of logistic regression and ROC curve analysis demonstrated that the predictive value of the questionnaire has sufficient accuracy in practice. When the adherence score obtained by patients with STI reaches 9 , we believe that the patient would perform well, with regular PrEP uptake and good adherence. The same methods for evaluating the predictive value of indicators have been used previously. ${ }^{30-33}$

Use of the PrEP adherence questionnaire provides the opportunity to evaluate patients' adherence in advance, such that a patient with low adherence can be detected prior to starting PrEP therapy. Individualbased measures can be used to improve adherence or to detect the need to use prevention measures other than PrEP, which could be more suitable for an individual and yield greater benefits. An improved PrEP adherence questionnaire considering more influencing factors has been greatly needed. 


\section{Limitations}

Owing to the fact that the test-retest reliability of the questionnaire was not measured during the reliability assessment, the integrity of the reliability assessment was reduced to some extent. In addition, we could not directly observe and track participants' drug use behavior in the real world, on the basis of which to determine their adherence. An additional question was asked to assess patients' behavioral intention as representing actual behavior; conservative prediction was adopted simultaneously to offset the uncertainty created during this process. The study population was not randomly sampled, resulting in insufficient sample representation, and the selected participants were limited to patients with STI. Owing to these limitations, whether our PrEP adherence questionnaire could be applicable to other populations and locations outside Shanghai is unknown. However, a total 816 participants were included in this research, which represents the population with STI in the survey area to some extent.

\section{Conclusion}

The reliability and validity of the PrEP adherence questionnaire is generally acceptable. The questionnaire includes four subscales: benefits, barriers, peer support, and self-efficacy, with two items for each. The present PrEP adherence questionnaire can be used as a tool to evaluate PrEP medication adherence in patients with STI.

\section{Abbreviations}

PrEP: pre-exposure prophylaxis

STI: sexually transmitted infection

ROC: receiver operating characteristic

CVI: content validity index

AIDS: acquired immunodeficiency syndrome

HIV: human immunodeficiency virus

VOICE trial: the Vaginal and Oral Interventions to Control the Epidemic trial

FEM-PrEP trial: the Preexposure Prophylaxis Trial for HIV Prevention among African Women

iPrEx trial: the Preexposure Prophylaxis Initiative

Partner PrEP Study: the Partners Preexposure Prophylaxis

UNAIDS: Joint United Nations Programme on HIV/AIDS 
I-CVI: item-level content validity index

S-CVI: scale-level content validity index

EFA: exploratory factor analysis

CFA: confirmatory factor analysis

KMO value: Kaiser-Meyer-Olkin value

df: degrees of freedom

RMSEA: root mean square error of approximation

GFI: goodness-of-fit index

AGFI: adjusted goodness-of-fit index

CFI: comparative fit index

AVE: average variance extracted

CR: construct reliability

AUC: the area under the receiver operating characteristic curve

\section{Declarations}

\section{Ethics approval and consent to participate}

The purpose and potential risks of this study were indicated in the informed consent forms presented to eligible participants, as was a guarantee of personal privacy. All consent forms were signed voluntarily by the included participants, who were informed of their right to opt out at any time during the research. Compensation of 80-yuan (about \$12) was provided to each survey respondent for their participation. During the entire process of dealing with participants, we strictly complied with the principles of the Declaration of Helsinki. This research obtained approval from the Shanghai Jiao Tong University School of Medicine Public Health and Nursing Ethics Committee (approval number: SJUPN-201702).

\section{Consent for publication}

Not applicable

\section{Availability of data and materials}


All data generated or analyzed during this study are included in this published article [and its supplementary information files].

\section{Competing interests}

The authors declare that they have no competing interests

\section{Funding}

The National Natural Science Funds of China $(71603166,71673187)$. The funders had no role in the design of the study and collection, analysis, and interpretation of data and in writing the manuscript

\section{Authors' contributions}

$X Y, C X$ and $Y C$ conceived the idea; $X Y$ conducted the analyses; $X Y$ wrote the initial draft of the paper; The remaining authors contributed to the writing and revisions.

\section{Acknowledgements}

We acknowledge all participants involved in collection of questionnaires. We thank Shanghai Skin Disease Hospital for coordinating data collection for patients with sexually transmitted infection and without HIV infection.

\section{References}

1. Riddell Jt, Amico KR, Mayer KH. HIV Preexposure Prophylaxis: A Review. Jama. 2018;319:1261-8. doi:10.1001/jama.2018.1917.

2. Wu Z, Chen J, Scott SR, McGoogan JM. History of the HIV Epidemic in China. Curr HIV/AIDS Rep. 2019;16:458-66. doi:10.1007/s11904-019-00471-4.

3. WHO. Guideline on when to start antiretroviral therapy and on pre-exposure prophylaxis for HIV, $<$ https://www.who.int/hiv/pub/guidelines/earlyrelease-arv/en/> (2015).

4. Baeten JM, et al. Antiretroviral prophylaxis for HIV prevention in heterosexual men and women. $N$ Engl J Med. 2012;367:399-410. doi:10.1056/NEJMoa1108524.

5. McCormack S, et al. Pre-exposure prophylaxis to prevent the acquisition of HIV-1 infection (PROUD): effectiveness results from the pilot phase of a pragmatic open-label randomised trial. Lancet. 2016;387:53-60. doi:10.1016/s0140-6736(15)00056-2.

6. Molina JM, et al. On-Demand Preexposure Prophylaxis in Men at High Risk for HIV-1 Infection. N Engl J Med. 2015;373:2237-46. doi:10.1056/NEJMoa1506273. 
7. Thigpen MC, et al. Antiretroviral preexposure prophylaxis for heterosexual HIV transmission in Botswana. N Engl J Med. 2012;367:423-34. doi:10.1056/NEJMoa1110711.

8. Marrazzo JM, et al. Tenofovir-based preexposure prophylaxis for HIV infection among African women. N Engl J Med. 2015;372:509-18. doi:10.1056/NEJMoa1402269.

9. Van Damme L, et al. Preexposure prophylaxis for HIV infection among African women. N Engl J Med. 2012;367:411-22. doi:10.1056/NEJMoa1202614.

10. Grant RM, et al. Preexposure chemoprophylaxis for HIV prevention in men who have sex with men. N Engl J Med. 2010;363:2587-99. doi:10.1056/NEJMoa1011205.

11. Desai M, Field N, Grant R, McCormack S. Recent advances in pre-exposure prophylaxis for HIV. Bmj. 2017;359:j5011. doi:10.1136/bmj.j5011.

12. Spinelli MA, Buchbinder SP. PrEP Persistence is a Critical Issue in PrEP Implementation. Clin Infect Dis. 2019. doi:10.1093/cid/ciz896.

13. Haberer JE. Current concepts for PrEP adherence in the PrEP revolution: from clinical trials to routine practice. Curr Opin HIV AIDS. 2016;11:10-7. doi:10.1097/coh.0000000000000220.

14. Wang Z, et al. Acceptability of Daily Use of Free Oral Pre-exposure Prophylaxis (PrEP) Among Transgender Women Sex Workers in Shenyang, China. AIDS Behav. 2017;21:3287-98. doi:10.1007/s10461-017-1869-4.

15. Golub SA, Gamarel KE, Rendina HJ, Surace A, Lelutiu-Weinberger CL. From efficacy to effectiveness: facilitators and barriers to PrEP acceptability and motivations for adherence among MSM and transgender women in New York City. AIDS Patient Care STDS. 2013;27:248-54. doi:10.1089/apc.2012.0419.

16. Gilmore HJ, et al. Participant experiences and facilitators and barriers to pill use among men who have sex with men in the iPrEx pre-exposure prophylaxis trial in San Francisco. AIDS Patient Care STDS. 2013;27:560-6. doi:10.1089/apc.2013.0116.

17. Grant RM, et al. Uptake of pre-exposure prophylaxis, sexual practices, and HIV incidence in men and transgender women who have sex with men: a cohort study. Lancet Infect Dis. 2014;14:820-9. doi:10.1016/s1473-3099(14)70847-3.

18. Pacífico de Carvalho, N, Mendicino CCP, Cândido RCF, Alecrim DJD. \& Menezes de Pádua, C. A. HIV pre-exposure prophylaxis (PrEP) awareness and acceptability among trans women: a review. AIDS Care. 2019;31:1234-40. doi:10.1080/09540121.2019.1612014.

19. Tangmunkongvorakul A, et al. Facilitators and barriers to medication adherence in an HIV prevention study among men who have sex with men in the iPrEx study in Chiang Mai, Thailand. AIDS Care. 2013;25:961-7. doi:10.1080/09540121.2012.748871.

20. Clement ME, et al. Advancing the HIV Pre-Exposure Prophylaxis Continuum: A Collaboration Between a Public Health Department and a Federally Qualified Health Center in the Southern United States. AIDS Patient Care STDS. 2019;33:366-71. doi:10.1089/apc.2019.0054.

21. Hoagland $B$, et al. High pre-exposure prophylaxis uptake and early adherence among men who have sex with men and transgender women at risk for HIV Infection: the PrEP Brasil demonstration project. 
J Int AIDS Soc. 2017;20:21472. doi:10.7448/ias.20.1.21472.

22. Lee PM, Ho KM. Risk factors associated with human immunodeficiency virus (HIV) infection among attendees of public sexually transmitted infection clinics in Hong Kong: implications for HIV prevention. Hong Kong Med J. 2008;14:259-66.

23. Galvin SR, Cohen MS. The role of sexually transmitted diseases in HIV transmission. Nat Rev Microbiol. 2004;2:33-42. doi:10.1038/nrmicro794.

24. Liu Z, et al. Changing epidemiological patterns of HIV and AIDS in China in the post-SARS era identified by the nationwide surveillance system. BMC Infect Dis. 2018;18:700. doi:10.1186/s12879018-3551-5.

25. UNAIDS. 90-90-90: Treatment for all, <http://www.unaids.org.cn/page147?_l=en\&article_id=986> (2020).

26. McEachan RRC, Conner M, Taylor NJ, Lawton RJ. Prospective prediction of health-related behaviours with the Theory of Planned Behaviour: a meta-analysis. Health Psychology Review. 2011;5:97-144. doi:10.1080/17437199.2010.521684.

27. Wood S, et al. Barriers and Facilitators of PrEP Adherence for Young Men and Transgender Women of Color. AIDS Behav. 2019;23:2719-29. doi:10.1007/s10461-019-02502-y.

28. Golub SA. PrEP Stigma: Implicit and Explicit Drivers of Disparity. Curr HIV/AIDS Rep. 2018;15:190-7. doi:10.1007/s11904-018-0385-0.

29. Van der Elst EM, et al. High acceptability of HIV pre-exposure prophylaxis but challenges in adherence and use: qualitative insights from a phase I trial of intermittent and daily PrEP in at-risk populations in Kenya. AIDS Behav. 2013;17:2162-72. doi:10.1007/s10461-012-0317-8.

30. Knutzen L, Anderson-Knight H, Svirko E, Impey L. Umbilical cord arterial base deficit and arterial pH as predictors of adverse outcomes among term neonates. Int J Gynaecol Obstet. 2018;142:66-70. doi:10.1002/ijgo.12502.

31. Lutz J, Gross RT, Vargovich AM. Difficulties in emotion regulation and chronic pain-related disability and opioid misuse. Addict Behav. 2018;87:200-5. doi:10.1016/j.addbeh.2018.07.018.

32. Mansbach WE, Mace RA. A comparison of the diagnostic accuracy of the AD8 and BCAT-SF in identifying dementia and mild cognitive impairment in long-term care residents. Neuropsychol Dev Cogn B Aging Neuropsychol Cogn. 2016;23:609-24. doi:10.1080/13825585.2016.1143443.

33. Tsushima WT, Geling O, Fabrigas J. Comparison of MMPI-2 validity scale scores of personal injury litigants and disability claimants. Clin Neuropsychol. 2011;25:1403-14. doi:10.1080/13854046.2011.613854.

\section{Figures}




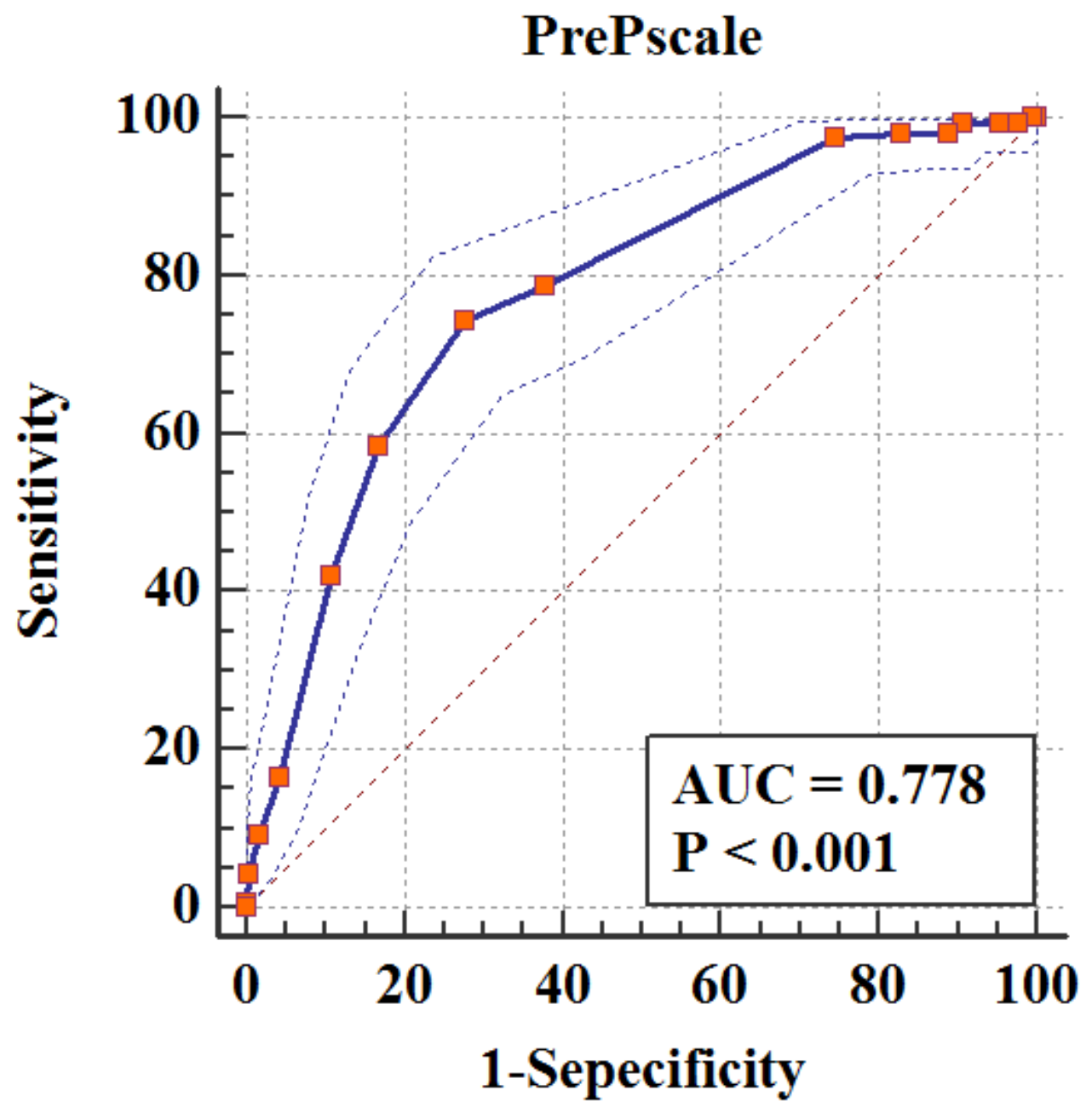

Figure 1

Receiver operating characteristic curve analysis of pre-exposure prophylaxis (PrEP) adherence questionnaire score for predicting PrEP uptake behavior

\section{Supplementary Files}

This is a list of supplementary files associated with this preprint. Click to download.

- Additionalfile1.docx 